\title{
COVID-19, Sistema Renina-Angiotensina, Enzima Conversora da Angiotensina 2 e Nicotina: Qual a Inter-Relação?
}

\author{
COVID-19, Renin-Angiotensin System, Angiotensin-Converting Enzyme 2, and Nicotine: What is the Interrelation?
}

\author{
Jaqueline Ribeiro Scholz, ${ }^{1 \oplus}$ Marcelo Antônio Cartaxo Queiroga Lopes, ${ }^{2,3,4}$ José Francisco Kerr Saraiva, 4,5๑ \\ Fernanda Consolim Colombo ${ }^{1}$ \\ Instituto do Coração do Hospital das Clinicas da Faculdade de Medicina da Universidade de São Paulo, ${ }^{1}$ São Paulo, SP - Brasil \\ Hospital Alberto Urquiza Wanderley - Hemodinâmica e Cardiologia Intervencionista, ${ }^{2}$ João Pessoa, PB - Brasil \\ Hospital Metropolitano Dom José Maria Pires, ${ }^{3}$ João Pessoa, PB - Brasil \\ Sociedade Brasileira de Cardiologia, ${ }^{4}$ Rio de Janeiro, RJ - Brasil \\ Pontifícia Universidade Católica de Campinas, ${ }^{5}$ Campinas, SP - Brasil
}

A Organização Mundial da Saúde (OMS) declarou a COVID-19, infecção causada pelo novo Coronavírus (SARS-CoV-2), ${ }^{1}$ como uma pandemia em 11 de março de 2020. Até o início do mês de junho, foram contabilizados 7 milhões de casos positivos e cerca de 400 mil mortes pela doença no mundo. ${ }^{2}$ No Brasil, nesse mesmo período, foram aproximadamente 700 mil casos e cerca de 40 mil óbitos. ${ }^{3}$

Embora o vírus possa infectar indivíduos de qualquer idade, até o momento, a maioria dos casos graves foi descrita naqueles com mais de 55 anos, com comorbidades associadas, muitas delas cardiovasculares. ${ }^{4,5}$ Portanto, justifica-se a grande preocupação da comunidade médica em saber como atuar frente à COVID-19, em especial nessa população de maior risco e com muitas comorbidades cardiovasculares, com o objetivo de reduzir as taxas de morbimortalidade. . $^{4}$

O SARS-CoV-2 usa como receptor de entrada na célula a enzima conversora de angiotensina tipo 2 (ECA-2), ${ }^{6}$ uma molécula expressa em abundância na superfície das células do endotélio, dos rins, dos pulmões e de outros órgãos. Ela é um componente do sistema renina-angiotensina (SRA), cuja sequência genômica foi descoberta em 2000. ${ }^{6}$ A partir de então, foi possível reconhecer um eixo compensatório das ações clássicas do SRA (eixo "protetor") para contrapor o eixo deletério causado pela produção da angiotensina 2. Do ponto de vista estrutural, a ECA-2 é semelhante à clássica; porém, do ponto de vista funcional, elas se contrapõem. ${ }^{7}$ Isso porque a ECA converte a angiotensina $1 \mathrm{em}$ angiotensina 2 e provoca efeitos deletérios decorrentes da estimulação dos receptores

\section{Palavras-chave}

COVID-19; Coronavirus/complicações; Betacoronavirus, SARS-CoV2; Syndrome Respiratory Acute; SARS-CoV2.

Correspondência: Jaqueline Ribeiro Scholz •

Universidade de São Paulo Instituto do Coração - Rua Dr. Eneas de Carvalho Aguair, $441^{\circ}$ andar, bloco 2. CEP 05403-900, São Paulo, SP - Brasil E-mail: jaquelineincor@yahoo.com.br

Artigo recebido em 14/06/2020, revisado em 24/06/2020, aceito em $24 / 06 / 2020$

DOI: https://doi.org/10.36660/abc.20200653
AT1, como aumento da atividade simpática, reabsorção de sal e água, vasoconstrição, inflamação, liberação de aldosterona e vasopressina, contribuindo para fibrose tecidual, disfunção do endotélio e hipertensão arterial. A ECA-2 decompõe a angiotensina 2 em seus metabólitos, incluindo angiotensina (1 a 9) e angiotensina (1 a 7), e ativa receptores mas, que são potentes vasodilatadores e, portanto, podem ser um regulador negativo do SRA. ${ }^{7}$ A ECA-2 é expressa em uma variedade de tecidos diferentes, incluindo as vias respiratórias superiores e inferiores, o miocárdio e a mucosa gastrointestinal. ${ }^{8}$ Embora sua função na saúde e na doença humana não tenha sido totalmente elucidado, ela parece ter um importante papel regulador na pressão sanguínea e na função cardíaca. O papel fisiológico da ECA-2 nas vias respiratórias é ainda desconhecido; no entanto, em camundongos, foi demonstrado que ela protege de lesões pulmonares graves relacionadas a aspiração e sepse. ${ }^{9}$

As questões envolvendo a relação entre maior disponibilidade de receptores de ECA-2 e, possivelmente, maior susceptibilidade para a infecção pelo SARS-Cov- $2^{2}$ são amplamente debatidas na cardiologia, visto que o uso de medicamentos como inibidores da enzima conversora de angiotensina (IECA) e bloqueadores do receptor de angiotensina 2 (BRA) aumenta a expressão dos receptores da ECA-2 em diferentes tecidos, incluindo o pulmão, ${ }^{10}$ embora seja fundamental para o tratamento da hipertensão arterial e insuficiência cardíaca. ${ }^{11,12}$ Houve discussões a respeito da substituição desses fármacos na vigência da pandemia; porém, devido à relevância no quesito eficácia e segurança no tratamento das doenças cardiovasculares e, até o momento, à ausência de evidências da relação entre o uso deles e o aumento da mortalidade pela COVID-19, há um consenso ${ }^{13}$ quanto à manutenção dos mesmos até haver evidências robustas que possam indicar o contrário. Na verdade, a boa notícia é que os estudos sugerem até mesmo um efeito protetor dos IECA na redução de mortalidade durante infecção pelo SARSCoV-2, e nenhuma comprovação de aumento de risco em usuários de BRA. ${ }^{14}$

É interessante observar que outro aspecto muito relevante e polêmico também envolve a expressão da ECA-2 e se relaciona ao tabagismo. Alguns autores ${ }^{15,16}$ levantaram a hipótese de que a observação da baixa 
prevalência de fumantes internados com COVID-19 na China e na França, em comparação com a prevalência mais elevada do tabagismo na população geral, possa ter relação com a menor expressão de ECA-2, provocada pela nicotina. ${ }^{17}$ Oakes et al. ${ }^{17}$ fazendo uma revisão sobre efeitos da nicotina e SRA, demostrou que a nicotina inalada altera a homeostase do SRA pulmonar, por estimular seu eixo clássico (aumento de expressão e concentração de ECA-ANG 2) em detrimento do eixo protetor (redução de expressão e concentração de ECA-2 e angiotensina 1-7), determinando, assim, menor expressão de ECA-2. Desse modo, os defensores da hipótese ${ }^{16}$ do efeito "protetivo" da nicotina especulam que isso dificultaria a adesão do SARS-Cov-2 no epitélio respiratório. Cabe ressaltar que a média de idade dos pacientes internados com COVID-19 é mais elevada, ${ }^{4}$ e a prevalência do tabagismo cai significativamente com o envelhecimento, tanto porque fumantes morrem precocemente ${ }^{18}$ quanto porque deixam de fumar quando adoecem. ${ }^{18}$ Novamente, esse é um paradoxo envolvendo a expressão dos receptores de ECA-2 e o SRA.

Nesse contexto, algumas perguntas devem ser respondidas: existem dados epidemiológicos que indiquem esse efeito "protetor"? Qual é a ação da nicotina sobre o SRA no epitélio brônquico? A relação entre expressão de ECA-2 no epitélio pulmonar é semelhante entre fumantes e não fumantes? Quais as consequências da interrupção na homeostase do SRA no pulmão causada pela nicotina?

Os dados de mortalidade mostram maior risco de morte por COVID-19 em fumantes com ou sem doença pulmonar obstrutiva crônica (DPOC), ${ }^{19-21}$ e o risco de intubação é dobrado $^{19}$ comparando-se fumantes com não fumantes. Esses dados corroboram o que ocorre em outras infecções virais, cujo curso é pior em fumantes. ${ }^{22,23}$ Considerandose a complexidade do SRA, a nicotina pode afetar outros elementos além dos discutidos, provocando efeitos ainda não elucidados.

Estudos recentes demonstraram aumento da expressão da ECA-2 no epitélio de pequenas vias respiratórias de tabagistas e portadores de DPOC com COVID-19. Brake et al., ${ }^{24}$ por meio da imuno-histoquímica, identificaram pela primeira vez uma expressão aumentada de ECA-2 no tecido pulmonar de pacientes com COVID-19. Porém ela foi maior nos pacientes com DPOC, fumantes ou não, e em menor proporção em fumantes sem DPOC. Não se encontrou aumento da expressão de ECA-2 em indivíduos não fumantes. Leung et al. ${ }^{25}$ também observaram maior expressão da ECA-2 no epitélio das pequenas vias respiratórias de pacientes com DPOC e fumantes com COVID-19, analisando material de lavado brônquico e correlacionando isso à gravidade da doença. Russo et al. ${ }^{26}$ investigaram in vitro o mecanismo pelo qual a nicotina poderia levar ao aumento da ECA-2 nessa população. Diferentes células das vias respiratórias, como epiteliais brônquicas, epiteliais alveolares tipo 2 e fibroblastos intersticiais, expressam receptores nicotínicos de acetilcolina (nAChR), especificamente o subtipo a7-nAChR, e também os componentes do SRA. Quantificando-se a expressão de ECA-2 em células epiteliais brônquicas em cultura, foi possível demonstrar que a nicotina promove regulação positiva (aumento da expressão de ECA-2) mediada especificamente pela sua ligação com os receptores a7-nAChR. Assim, o tabagismo poderia provocar um aumento no mecanismo de captação celular para o SARS-Cov-2 por meio da sinalização da via $a 7-n A C h R$.

Com esses dados, o raciocínio seria de que os pacientes tabagistas e com DPOC teriam, na verdade, mais susceptibilidade à infecção pelo SARS-Cov-2. Esse mecanismo, inclusive, foi formulado e representado em modelo esquemático (Figura 1) por Olds e Kabbani ${ }^{27}$ e explica como a exposição à nicotina aumenta o risco de entrada do vírus nas células pulmonares e, consequentemente, como o ato de fumar pode ter impacto negativo na fisiopatologia da COVID-19.

Nesse contexto, pode-se interpretar que o papel do SRA na gravidade da infeção por SARS-CoV-2 depende menos da expressão da ECA-2 no aparelho cardiovascular e mais da expressão dele no epitélio respiratório. Isso pode justificar a não interferência na morbimortalidade por COVID-19 em usuários de IECA e BRA, bem como a falta de proteção para essa doença em tabagistas e pacientes com DPOC.

Além de as orientações terapêuticas serem parar de fumar e manter a medicação cardiovascular, o conhecimento da inter-relação da nicotina com a expressão de ECA-2 nas células do epitélio respiratório e sua interface com receptores de a7-nAChR sugere a possibilidade de ações terapêuticas para tratamento da COVID 19. O uso de antagonistas seletivos de a7-nAChR, como metilglicaconitina ${ }^{28}$ e a-conotoxina, ${ }^{29}$ pode alterar significativamente a expressão de ECA-2, podendo ser uma opção terapêutica para impedir a entrada de SARS-CoV-2 no epitélio das vias respiratórias. Futuros estudos deverão confirmar ou não essas hipóteses.

\section{Contribuição dos autores}

Concepção e desenho da pesquisa e Obtenção de dados: Scholz JR; Análise e interpretação dos dados e Redação do manuscrito: Scholz JR, Consolim F; Revisão crítica do manuscrito quanto ao conteúdo intelectual importante: Lopes MACQ, Saraiva JFK, Consolim F.

\section{Potencial conflito de interesses}

Declaro não haver conflito de interesses pertinentes.

\section{Fontes de financiamento}

O presente estudo não teve fontes de financiamento externas.

\section{Vinculação acadêmica}

Não há vinculação deste estudo a programas de pósgraduação. 


\section{Carta Científica}

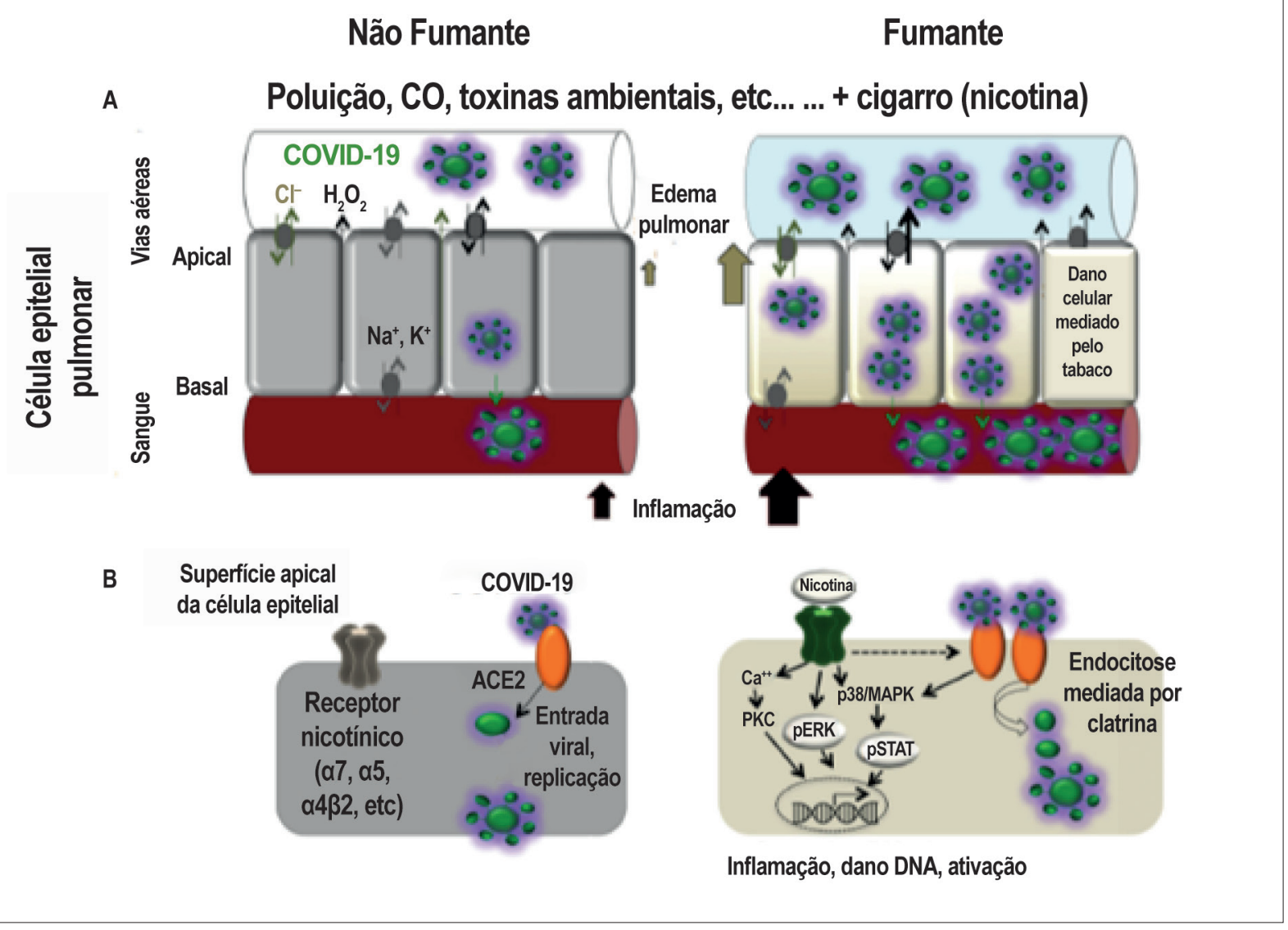

Figura 1 - Modelo esquemático de como a exposição à nicotina aumenta o risco de entrada do SARS-CoV-2 no pulmão do hospedeiro humano. A. Respostas pulmonares e imunes à infecção pelo vírus em células epiteliais de fumantes (à direita) e não fumantes (à esquerda). B. Mecanismos celulares desencadeados pela atividade de receptores nicotínicos promovem a entrada e proliferação do SARS-CoV-2 nas células epiteliais por meio da coexpressão da ECA-2. A ativação de receptores nicotínicos pela nicotina pode causar maior ativação de proteases, morte celular (apoptose) e sinalização inflamatória por meio de mecanismos que convergem nas vias de regulação e sinalização da ECA-2.

\section{Referências}

1. World Health Organization. Coronavirus disease (COVID-19) outbreak. Disponível em: www.who.int/emergencies/diseases/novelcoronavirus-2019/events-as-they-happen.

2. Max Roser, Hannah Ritchie, Esteban Ortiz-Ospina et al. Coronavirus pandemic (COVID-19). [Cited in 2020 Apr 20]. Available from: https:// ourworldindata.org/coronavirus.

3. Brasil. Ministério da Saúde. [Acesso em 03 jun 2020]. Disponível em: https://covid.saude.gov.br.

4. Zhou P, Yang XL, Wang XG, Hu B, Zhang L, Zhang W, et al. A pneumonia outbreak associated with a new coronavirus of probable bat origin. Nature. 2020; 579(7798):270-3.

5. Wu Z, McGoogan JM. Characteristics of and important lessons from the coronavirus disease 2019 (Covid-19) outbreak in China: summary of a report of 72314 cases from the chinese Center for Disease Control and prevention. J AmMed Assoc. 2020;323(13):1239-42.

6. Donoghue M, Hsieh F, Baronas E, Godbout K, Gosselin M, Stagliano N, et al. A novel angiotensin-converting enzyme-related carboxypeptidase (ECA-2) converts angiotensin i to angiotensin 1-9. Circ Res. 2000; 87(5):e1-e9.
7. Crackower MA, Sarao R, Oudit GY Yagil C, Kozieradzki I, Scanga SE, et al. Angiotensin-converting enzyme 2 is an essential regulator of heart function. Nature. 2002; 417(6891):822-8.

8. Harmer D, Gilbert M, Borman R Clark KL. Quantitative mRNA expression profiling of ACE 2, a novel homologue of angiotensin converting enzyme. FEBS Lett. 2002; 532(1-2):107-10.

9. Imai Y, Kuba K, Rao S Huan Y, Guo F, Guan B, et al. Angiotensinconverting enzyme 2 protects from severe acute lung failure. Nature. 2005; 436(7047):112-6.

10. Campbell DJ. The site of angiotensin production. J Hypertens. 1985; 3(3):199-207.

11. Moser M. Angiotensin-converting enzyme inhibitors, angiotensin II receptor antagonists and calcium channel blocking agents: a review of potential benefits and possible adverse reactions. J Am Coll Cardiol. 1997; 29(7):1414-21. Disponível em: https://doi.org/10.1016/S07351097(97)00096-X.

12. Li ECK, Heran BS, Wright JM. Angiotensin converting enzyme (ACE) inhibitors versus angiotensin receptor blockers for primary hypertension. Cochrane Database of Systematic Reviews. 2014; Issue 8. Art. n.: CD009096. 
13. Queiroga M, Bacal F, Hajjar LA. Infecção pelo Coronavírus 2019 (COVID-19). Disponível em: http://www.cardiol.br/sbcinforma/2020/20200313comunicado-coronavirus.html. 2020.

14. Richardson S, Hirsch JS, Narasimhan M Crawford JM, McGinn T, Davidson KW, et al. Presenting characteristics, comorbidities, and outcomes among 5700 patients hospitalized with COVID-19 in the New York City area. JAMA. 2020; 323(20):2052-9.

15. Simons D, Shahab L, Brown J,Perski O. The association of smoking status with SARS-CoV-2 infection, hospitalization and mortality from COVID-19: a living rapid evidence review. Qeios. 2020.[Cited in 2020 May 23] Available from: qeios.com/read/UJR2AW2

16. Farsalinos K, Barbouni A, Niaura R. Systematic review of the prevalence of current smoking among hospitalized COVID-19 patients in China: could nicotine be a therapeutic option? Intern Emerg Med. 2020; 15(5):845-52.

17. Oakes JM, Fuchs RM, Gardner JD et al. Nicotine and the renin-angiotensin system. Am J Physiol Regul Integr Comp Physiol. 2018; 315(5):R895-R906.

18. Doll R, Peto R, Boreham J et al. Mortality in relation to smoking: 50 years' observations on male British doctors. BMJ. 2004; 328(7455):1519.

19. Zhao Q, Meng M, Kumar R Wu Y, Huang J, Lian N, et al. The impact of COPD and smoking history on the severity of COVID-19: a systemic review and meta-analysis doi: 10.1002/jmv.25889

20. Alqahtani JS, Oyelade T, Aldhahir AM et al. Prevalence, severity and mortality associated with COPD and smoking in patients with COVID-19: a rapid systematic review and meta-analysis. PLoS One. 2020; 15(5):e0233147.

21. Patanavanich R, Glantz SA. Smoking is associated with COVID-19 progression: a meta-analysis. Nicotine Tob Res 2020; doi 10.1093/ntr/ntaa082
22. WHO Framework Convention on TobaccControl. Increased risk of COVID-19 infection amongstsmokers and amongst waterpipe users. Disponível em: https:// untobaccocontrol.org/kh/waterpipes/covid-19. Acesso em: 2 mai 2020.

23. Alraddadi BM, Watson JT, Almarashi A Abedi GR, Turkistani A, Sadran M, et al. Risk factors for primary middle east respiratory syndrome coronavirus illness in humans, Saudi Arabia, 2014. Emerg Infect Dis. 2016; 22(1):49-55.

24. Brake SJ, Barnsley K, Lu W et al. Smoking upregulates angiotensin-converting enzyme-2 receptor: a potential adhesion site for novel Coronavirus SARSCoV-2 (Cvid-19). J Clin Med. 2020; 9(3):841.

25. Leung JM, Yang CX, Tam A Shaipanich T, Hackett TL, Singhera GK, et al. ACE2 expression in the small airway epithelia of smokers and COPD Patients: implications for COVID-19. Eur Resp J. 2020; 55(5): 2000688

26. Russo PB, Giacconi R, Malavolta M et al. COVID-19 and smoking. Is nicotine the hidden link? Eur Resp Jour. 2020;55(6); 2001116.doi: 10.1183/13993003.01116.2020.

27. Olds JL, Kabbani N. Is nicotine exposure linked to cardiopulmonary vulnerability to COVID-19 in the general population? FEBS J. 2020.[Cited in 2020 Apr 20]. doi: 10.1111/febs.15303.

28. Panagis G, Kastellakis A, Spyraki C Nomikos G. Effects of methyllycaconitine (MLA), an alpha 7 nicotinic receptor antagonist, on nicotine- and cocaineinduced potentiation of brain stimulation reward. Psychopharmacology (Berl). 2000; 149(4):388-96.

29. Liang J, Tae HS, Xu X Jiang T, Adams DJ, Yu R, et al. Dimerization of alphaconotoxins as a strategy to enhance the inhibition of the human alpha 7 and alpha 9, alpha 10 nicotinic acetylcholine receptors. J Med Chem. 2020; 63(6):2974-85 